\title{
Precision Spectroscopic Measurements in Few-Electron Ions
}

R. W. Dunford, H. G. Berry, D. A. Church,* T. P. Dinneen, M. Hass, $\dagger$ C. J. Liu, N. Berrah-Mansour, R. C. Pardo, M. L. A. Raphaelian, L. Young, and B. J. Zabransky

Physics Division, Argonne National Laboratory, Argonne Il 60439

\section{J. Curtis}

Department of Physics and Astronomy, University of Toledo, Toledo, $\mathrm{OH} 43606$

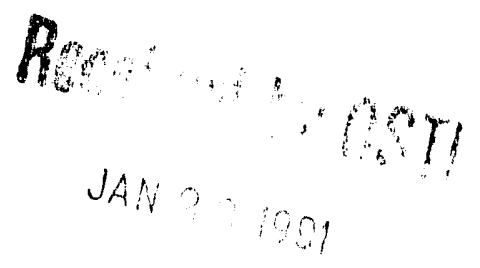

\section{DISCLAIMER}

This report was prepared as an account of work sponsored by an agency of the United States Government. Neither the United States Government nor any agency thereof, nor any of their employees, makes al:" warranty, express or implied, or assumes any legal liability or responsibility for the accuracy, completeness, or usefulness of any information, apparatus, product, or process disclosed, or represents that its use would nict infringe privately owned rights. Reference herein to any specific commercial product, process, or service by trade name, trademark, manufacturer, or otherwise does not necessarily constitute or imply its endorsement, recommendation, or favoring by the United States Government or any agency thereof. The views and opinions of authors exp. essed herein do not necessarily state or reflect those of the United States Government or any agency thereof.

\section{MASTER}


Abstract

We describe recent precision experiments in fewelectron ions including measurements of the lifetimes of two-photon-emitting levels in $\mathrm{Ni}^{26+}$ and $\mathrm{Ni}^{27+}$, a measurement of the lifetime of the ${ }^{3} \mathrm{~S}_{1}$ leve $\mathrm{I}$ in $\mathrm{Br}^{33+}$ and measurements of the $2^{3} \mathrm{~S}_{1}$. $2^{3} \mathrm{P}_{0,1,2}$ transition energies in $\mathrm{B}^{3+}$.

\section{Introduction}

Few-electron systems, particularly hydrogen-like and helium-like ions provide an important testing ground for our understanding of atomic structure. In the one-electron systens, there are no calculational uncertainties, so experiments in these systems provide tests of the underlying theory of Quantum Electrodynamics (QED). Current interest is in the question of whether there is a breakdown of QED in highly-charged one-electron atoms. The theory of the two-electron system is not as well understood. Here one has the additional problem of treating correlations between the two electrons and a complete Hamiltonian for the system does rot exist [1]. The importance of the helium-like systems is that they are the simplest atous in which the electronelectron correlations must be taken into $r .$. unt.

In this paper we cescribe four recent experiments done by our group which study the structure of one- and two-electron ions. The first two experiments are measurements of the lifetimes of the $2^{2} \mathrm{~s}_{1 / 2}$ level in one electron $\mathrm{Ni}^{2.7+}$ and the $2^{2} \mathrm{~S}_{0}$ level in two-electron $\mathrm{Ni}^{26+}$, both of which decay primarily by two-photon emission. The third experiment is a measurement of the lifetime of the $2^{3} \mathrm{~S}_{1}$ level in helium-like $\mathrm{Br}^{33+}$, which decays to the ground state via a Ml transition. The final experiment is precision measurement of the $2^{3} \mathrm{~s}_{1}$ - $2^{3} p_{0}, 2$ transition energies in two-electron $\mathrm{B}^{3+}$. This experiment was done by driving resonances in a $f_{a s t}$ beam of metastable $B^{3+}$ using a frequency doubled dye laser.

\footnotetext{
* Present address: Texas A\&M University, College Station, TX 778.43 .
}

†Present address: University of Rochester, Rochester, NY 1462.7 .

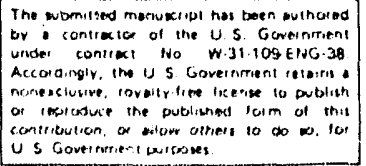

II. Two-Photon Decays in One- and Two-Electron Nickel

The ${ }^{1} \mathrm{~S}_{0}$ level. (see Fig. 1) in helium-like ions is forbidden to decay to the liso ground state by the emission of a single photon and so it decays via two-photon emission. The $2^{2} \mathrm{~s}_{1 / 2}$ level in hydrogen-like ions also decays to the ground state via two-photon emission although there is also a small MI branch $\left(17 \%\right.$ in $\left.\mathrm{Ni}^{27+}\right)$. We have measured the lifetimes of these two-photon emitting levels in orie- and two-electron nickel [2]. Our technique is to measure the rate of two-photon coincidences as the distance between our detectors and the beam-foil is varied.

ANI.P. 19,734

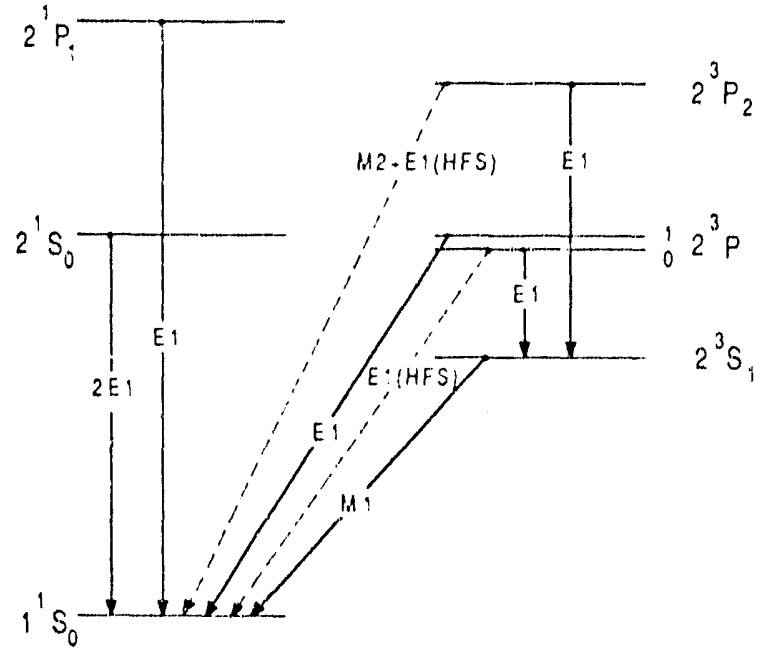

Fig. 1. Energy levels and decay modes for helium-1lke ions.

The data were taken using nickel beams from the Argonne Tandem-Linac (ATLAS). After acceleration, the ions were stripped in a thick carbon foil and the $26+$ or the $28+$ charge state was directed to the target area. The beam energy was measured by a time-of-flight energy analyzer and corrected for energy loss in the stripper foils [3]. In the carget area, a thin carbon target (12 $\left.\mu \mathrm{g} / \mathrm{cm}^{2}\right)$ is 
moved relative to three fixed $S i(L i)$ detectors using a precision translation stage. Two of the $S i(L i)$ detectors are collimated so they observe a region of length 5 man along the beam path and subtend a solid angle of 0.12 at the beam. The third detector is not highly collimated and accepts photons into a solid angle of 12 . There is also a lower resolution silicon X-ray detector attached to the target holder which is used for normalization.

Events were recorded for coincidences between any two of the three Si(Li) detectors, but most of the coincidences are between one of the collimated detectors and the large solid-angle detector. The collimators serve to localize the events in position along the beam while the large solidangle detector provides a good efficiency for detecting the second photon. In Fig. 2 we show a typical singles spectrum for one of the $S i(L i)$ detectors for the case of $\mathrm{Ni}^{26+}$ incident on the target.. The broad continuum extending out to 7.8 $\mathrm{keV}$ is mostly due to the decay of the $2^{1} 5_{0}$ level in helium-like $\mathrm{Ni}^{26+}$. In Fig. 3 we show the sumenergy spec'rum for true coincidences. In this case the sum of the energies of the two photons forms a peak at the transition energy. This peak is almost entirely from the helium-like two-photon decay. The spectrum taken with the $28+$ beam is predominantly from the two-photon decay of hydrogen-like nickel but there is a simall contamination from the helium-like sum energy peak which lies $300 \mathrm{eV}$ lower in energy.

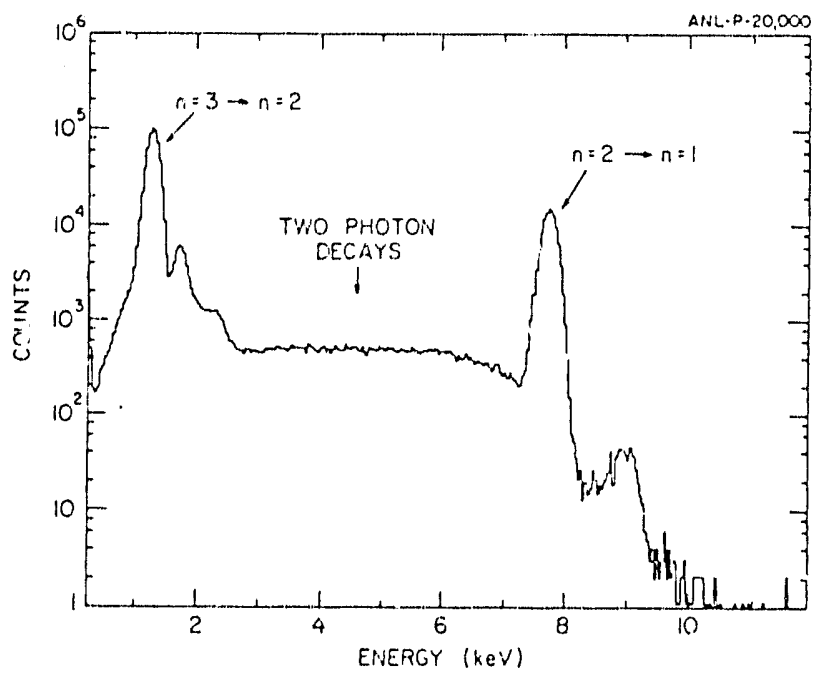

Fig. 2. Typical spectrum in the Si(Li) detector for helium-like $\mathrm{Ni}^{26+}$ ions incident on the target.

The data are normalized using the count from the nomalization detector and the decay curve is $f$ it to a function with two paramuters: a) the amplitude at the closest position and b) the lifetime. The lifetimes from these two component fits for each of the three detector pairs are combined and then corrected for time dilation to obtain the final result. A number of additional systematic effects were considered but found to be unimportant for these measurements including gain

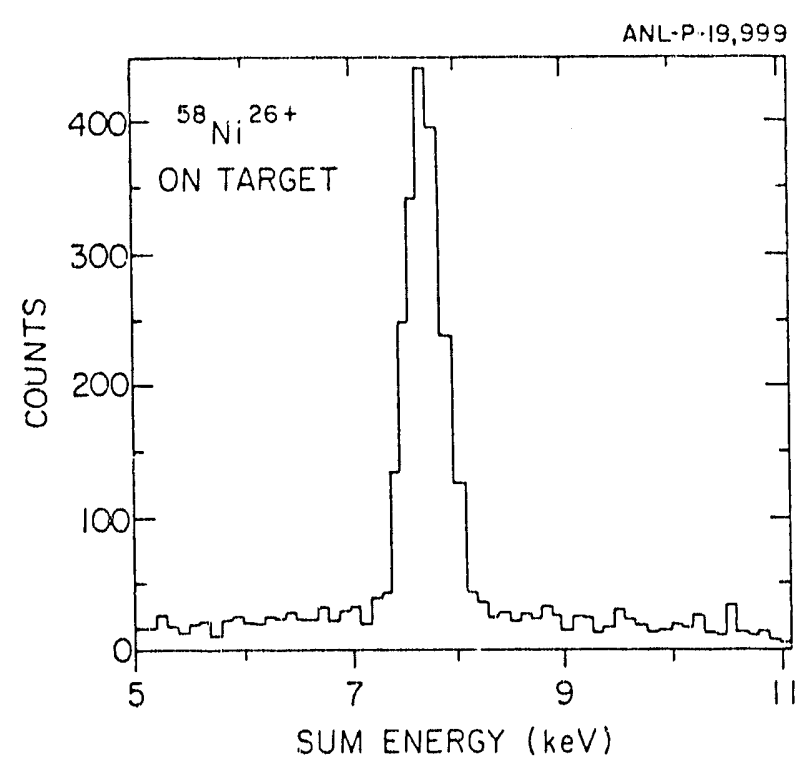

Fig. 3. Sum-energy spectrum for true coincidences with $\mathrm{Ni}^{26+}$ incident on the target.

shifts, quenching in electric and magnetic fields, contributions from ions with high-lying spectator electrons [4] and backgrounds from coincidences caused by Compton scattering of Lyman- $\alpha$ photons between two of the $S i(L i)$ detectors.

Two separate measurentunts were made for each of the two lifetimes. In each case, the measurements were consistent within errors and we simply combined them. For the helium-like decay the combined result is $156.1(1.6) \mathrm{ps}$ which is in substantial agreement with the theoretical (5) value of $154.3(0.5)$ ps. For the decay of the $2^{2} \mathrm{~S}_{1 / 2}$ state in hydrogen-like $\mathrm{Ni}^{27+}$ we find a lifetime of $217.1(1.8)$ ps which agrees with the theoretical value [6] of $2.15 .45 \mathrm{ps}$.

III. MI Decay of the $2^{3} \mathrm{~S}_{1}$ level ${ }^{79} \mathrm{Br}^{33+}$

The same apparatus has been used to measure the lifetime [7] of the $2^{3} \mathrm{~s}_{1}$ lipvel in helium-like $79 \mathrm{Br}^{33+}$. This level decays to the $I^{1} \mathrm{~S}_{0}$ ground state by a furlidden MI transition. Bromine-79 is a good choice for the measirement of the lifetime of the hellum-like $2^{3} \mathrm{~s}_{1}$ level because, in this isotope, all of the other $n=2$ levels which decay by single-photon emission are tnuch shorter lived and so there is a mininum of cumplication from the decay of other levels. An impurtant factor here is that the ${ }^{3} \mathrm{p}_{0}$ level is quenched to the ground state by hyperfine mixing induced by the magnetic moment of $79 \mathrm{Br}(I=3 / 2)$. This reduces the lifetime of this level from $1.48 \mathrm{~ns}$ (unquenched) to $5.6 \mathrm{ps}$ (quenched).

The bromine beam was provided by the Argonne Tandem-Linac (ATLAS). The hellum-like $33+$ charge state is ananetically analyzed and directed to the experimental area. Two detectors were used for this experiment and they were collimated so that they observe a region $2 \mathrm{~mm}$ along the beam. 
At each foil-detector separation, peaks near 12 $\mathrm{keV}$ in the $S i(L i)$ detectors, which correspond to single-photon decays from the $n=2$ levels of $\mathrm{Br}^{33+}$ were fitted to a Gaussian line shape. The peak areas at each distance were divided by the count for the notmalization detector and these data were fitted to a decay curve to obtain the lifetime. our final result is $T\left(2^{3} S_{1}\right)=224.1(7.1)$ ps, which agrees with the calculation by Drake [8] which gives $230(2)$ ps and the calculation by Johnson and Lin [9] which gives 228.2 ps.

\section{Laser Spectroscopy}

Spectroscopic measurements in helium-like ions include high-precision measurements in helium [10] and Iithium [11] which test the nonrelativistic parts of the interaction, and lower precision measurements in heavy ions which test the relativistic and QED corrections. A complete 1 ist of recent experimenis is given by Drake [12] along with his most recent calculations. The measurements in lithium test relativistic corrections to higher precision than measurements in helium but are limited at present by uncertainties in the nonrelativistic calculations of the energy. We have recently completed precision laser measurements of the $2 \mathrm{~s}$ to $2 \mathrm{p}$ transitions in $\mathrm{B}^{3+}$ where the relativistic and QED corrections are a larger fraction of the transition energy so they can be tested with less uncertainty arising from the nonrelativistic contribution to the calculations.

Metastable $\mathrm{B}^{3+}$ ions were produced in the Positive Ion Injector Electron Cyclotron Resunance (PIIECR) source at ATLAS. The source was operated at $10 \mathrm{kV}$ extraction voltage. Beam currents in the interaction region varied from 0.5 to $1.5 \mu \mathrm{A}$. Transitions from $2^{3} \mathrm{~S}_{1}$ to $2^{3} \mathrm{P}_{0}, 1,2$ were driveu by $282 \mathrm{~nm}$ laser light produced by intra-cavity frequency doubling of the $564 \mathrm{~nm}$ fundamental of a Coherent 699 ring dye laser. The maximum doubled output obtained froun the dye laser was $7 \mathrm{mil}$.

The ion beam and laser beam were overlapped to take advantage of the increased interaction time and the velocity compression provided by the accelerated beam. The Doppler width (FWHM) was 1 $\mathrm{GHz}$, corresponding to a beam energy spread of 21 eV. The transitions were monitored by detecting the fluorescence radiation emitted as the $2^{3} \mathrm{p}$ levels decay back to the $2^{3} s_{1}$ level. The sensitivity of the experiment was sufficient to allow most of the hyperfine components of $11_{B}{ }^{3+}$ (nuclear spin $I=3 / 2$ ) to be observed. In order to make the Doppler correction, the laser light was reflected back through the Interaction region and the laser was scanned over both the red and the blue Doppler-shifted components while simultaneously measuring an lodine absorption spectrum with the fundamental output of the dye laser. After making an absolute wavelength determination for one of the $2^{3} \mathrm{p}_{2}$ hyperfine transitions, all other transitions were measured relative to it.

To obtain hyperfine-free wavelengths it is necessary to measure all of the hyperfine components. Since the laser power stability was poor over large frequency scan ranges, a different technique was used to measure the hyperfine structure. This involved using the interaction region as a post acceleration section, and, keping the laser frequency fixed, the ion-beam velocity was tuned through the resonances by varying the interaction region voltage. In this way oll hyperfine components were obtained in a single spectrum as shown in Fig. 4 .

The experiment resulted in precision laser measurements of the fine structure, the hyperfine etructure and absolute wavelengths of the $(1 s 2 s)^{3} s_{1}$ to $(1 s 2 p)^{3} P_{0,1,2}$ transitions in heliumlike boron. These results are being prepared for publication [13]. The measurements provide a precision at the level of 0.12 of the one-electron Lamb shift for the $2 s+2 p$ transition.

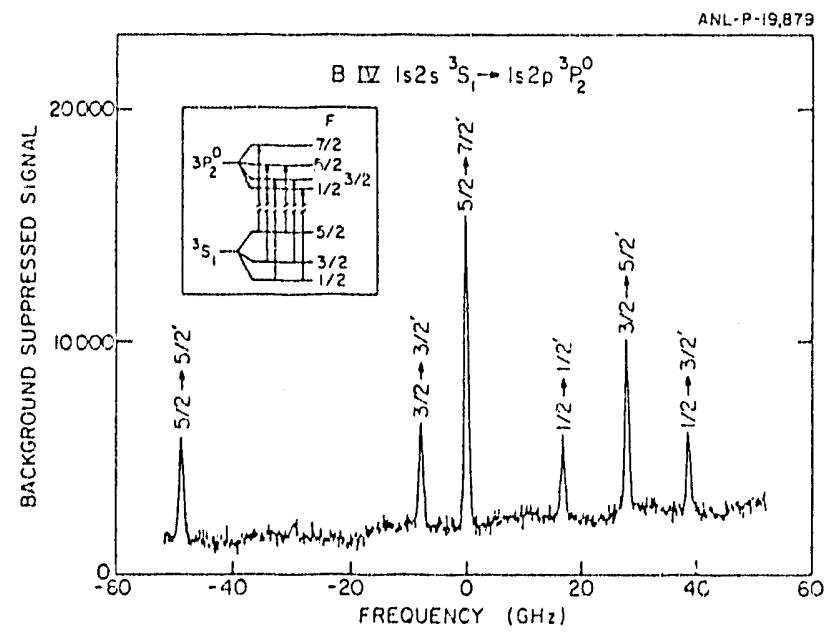

Fig. 4. Nearly complete hyperfine spectrum of the $2^{3} \mathrm{~S}_{1} \rightarrow 2^{3} \mathrm{P}_{2}$ transition in $\mathrm{B}^{3+}$. The resonances were scanned by varying the potential in the light collection region.

\section{v. Conclusion}

We have discussed a number of experiments that are sensitive to the relativistic and QED effects in one-and two-electron lons. The measurements of the lifetimes of the two- photon emitting states in $\mathrm{Ni}^{26+}$ and $\mathrm{Ni}^{27+}$ used the coincidence technique which resulted in simple-decay curves that could be fit with only two parameters. In the future we will apply the coincidence technique to more highly-charged one- and two-electron ions. The experiment to measure the lifetime of the $2^{3} \mathrm{~s}_{1}$ level in $79 \mathrm{Br}^{33+111 u s t r a t e s}$ the potential of atilizing the hyperfine quenching effect to reinove unwanted components from a blended line. We also described the first laser measurement of the $2^{3} \mathrm{~S}_{1}$ $+2^{3} P_{0,1,2}$ transition in the $B^{3+}$ system. With improved ion-beam intensity and metastable yield, there is potential for significant improvement in the precision of this experiment. We will also utilize this method to make measurements on other 
metastable helium-like ions which can be produced by our ECR ion source.

This work was supported by the U. S. Department of Energy, Office of Basic Energy Soiences, under Contract No. W-31-109-ENG-38.

\section{References:}

1. Lindgren, I., Nuc. Inst. Meth. Phys. Res. 31, 102 (1988).

2. Dunford, R. W., M. Hass, E. Bakke, H. G. Berry, C. J. Liu, and M. L. A. Raphaelian, L. J. Curtis, Phys. Rev. Lett. 62, 2809 (1989).

3. Ziegler, J. F., Handbook of Stopping Cross Sections for Energetic Ions in all Elements (Pergamon, New York, 1980).

4. Lin, D. L. and L. Armstrong, Phys. Rev. A 16 . 791 (1977).

๖. Drake, G. F. W., Phys. Rev. A 34, 2871 (1986).

6. Parpia, F. A. and W. R. Johnson, Phys. Rev. A 26. 1142 (1982).

7. Dunford, R. W., D. A. Church, C. J. Liu, H. G. Berry, M. L. A. Raphaelian, M. Hass, L. J. Curtis, Phys. Rev. A. 41, 4109 (1990).

8. Drake, G. W. F., Phys. Rev, A 3, 908 (1971); G. W. F. Drake (private comunication).

9. Johnson, W. R. and C. P. Lin, Phys. Rev. A 9 . 1486 (1974).

10. Zhao, P., J. R. Lawal1, A. W. Kam, M. D. Lindsay, F. M. Pipkin, W. Lichten Phys. Rev. Lett. 63, 1593 (1989).

11. Riis, E., H. G. Berry, O. Poulsen, S. A. Lee, S. Y. Tang, Phys, Rev, A 33,3023 (1986).

12. Drake, G. F. W., Can. J. Phys, 66, 586 (1988).

13. Dineen, T. P., N. B. Mansour, H. G. Berry, L. Young and $R$. C. Pardo, in preparation. 

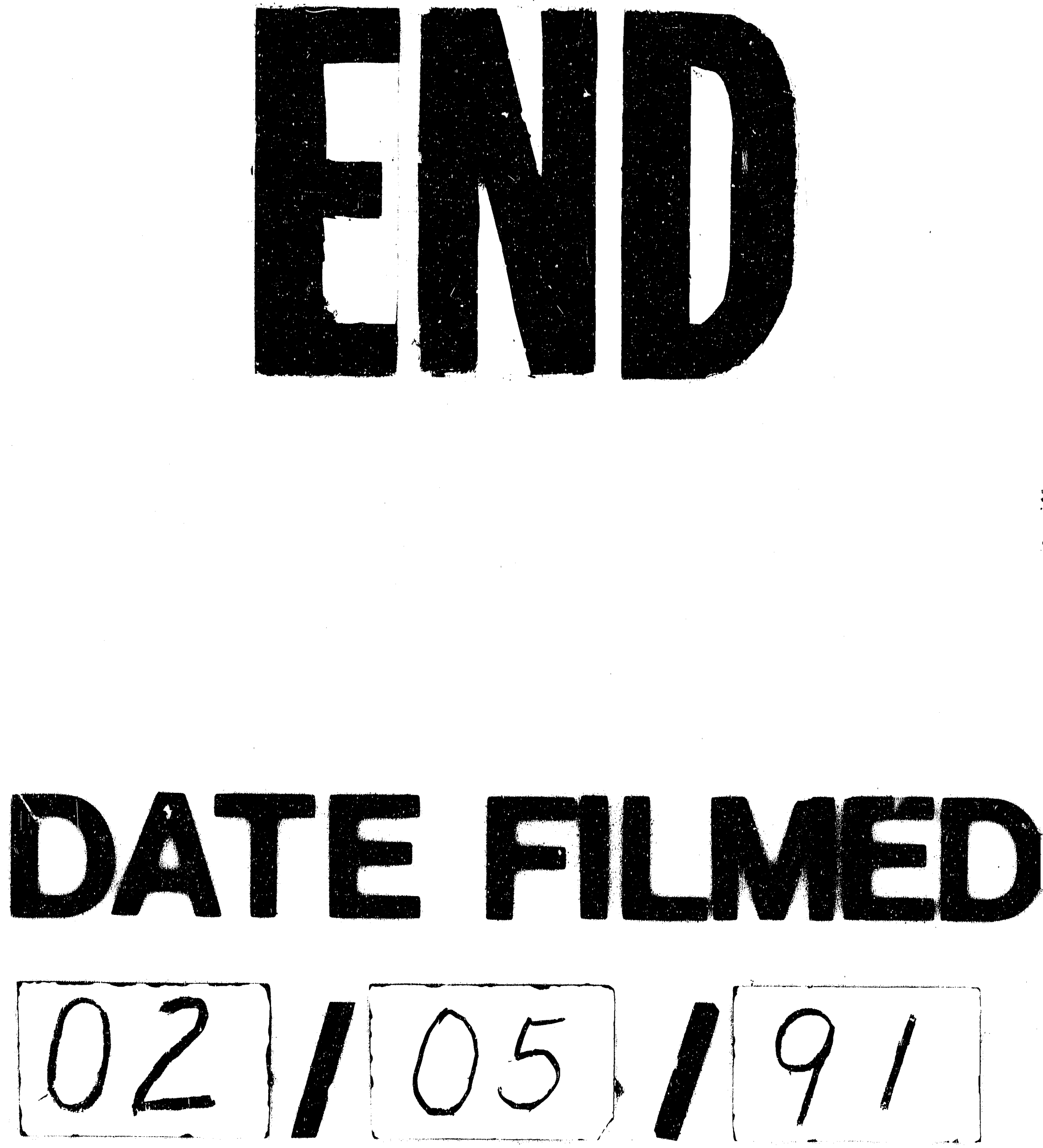
$\mid$ 